\title{
Localization and dynamic link detection for geographic routing in non-line-of-sight (NLOS) environments
}

\author{
Youngbae Kong ${ }^{1}$, Younggoo Kwon ${ }^{1 *}$, Jongho Shin ${ }^{1}$ and Gwitae Park ${ }^{2}$
}

\begin{abstract}
IEEE 802.15.4a networks can provide the geographic routing solution with high location accuracy for the indoor environments. However, in the non-line-of-sight (NLOS) environments, the IEEE 802.15.4a networks may have a large scale location error and an unstable communication link. In this article, we propose a location estimation and dynamic link detection scheme for the geographic routing in the NLOS environments. The proposed approach corrects the large scale location error and detects the NLOS link in the geographic routing procedure. Simulation and experimental results show that the proposed approach can enhance the performance of the geographic routing.
\end{abstract}

Keywords: geographic routing, IEEE 802.15.4a, NLOS environment, location estimation, link detection

\section{Introduction}

Wireless applications have been rapidly developed and its importance is continuously increasing. A geographic routing can provide the wireless applications with a flexible network solution. For example, when a wireless mobile device (also known as handheld device, smart phone) tries to exchange information (e.g., audio, image, or sensor data) with another device or a wired backbone network, it can download or upload the information throughout the wireless network. In that case, a geographic routing protocol can be attractive choice, since it can reduce the low route discovery overhead and needs only local neighbor information [1,2]. For the geographic routing in the wireless applications, mobile devices should obtain their physical locations by using a global positioning system (GPS) or localization techniques.

Recently, IEEE 802.15.4a Task Group (TG4) has developed two additional physical layers (PHYs) using ultrawideband (UWB) and chirp spread spectrum (CSS) [3]. The IEEE 802.15.4a enables high throughput communications with a precise ranging capability for low rate wireless personal area networks (LR-WPANs).

\footnotetext{
* Correspondence: ygkwon@konkuk.ac.kr

'Department of Electrical Engineering, Konkuk University, Seoul, Korea

Full list of author information is available at the end of the article
}

Therefore, the IEEE 802.15.4a networks can provide the geographic routing solution with high location accuracy for the indoor wireless applications, where a GPS does not operate.

Although the IEEE 802.15.4a provides the location solution for the geographic routing, it still has some difficulties in the indoor environments such as a company and a building hall. In the IEEE 802.15.4a, a ranging distance is determined through a two-way time-of-arrival (TW-TOA) technique, which calculates the distance by using the travel time of a radio signal $[4,5]$. In most cases, the indoor environments contain various objects, which cause a non-line-of-sight (NLOS) condition, and the ranging distance with TW-TOA may have the large measurement error in the NLOS condition. Through the experiments with IEEE 802.15.4a devices, we observed that in the NLOS condition, the mobile device has a large scale location error and a highly unstable wireless link. The location error and the unstable link may cause the performance degradation in the geographic routing. Moreover, since the current position of the mobile device is continuously changing, it is difficult for the mobile device to detect the NLOS link condition accurately. For the successful geographic routing in the NLOS environments, the mobile device should have

\section{SpringerOpen ${ }^{\circ}$}

(c) 2011 Kong et al; licensee Springer. This is an Open Access article distributed under the terms of the Creative Commons Attribution License (http://creativecommons.org/licenses/by/2.0), which permits unrestricted use, distribution, and reproduction in any medium, provided the original work is properly cited. 
capabilities to estimate its current location and to detect the wireless link condition accurately.

Several localization techniques have been proposed for indoor wireless applications [6-8]. However, their approaches are focused on the location accuracy and do not consider the effects of the location error in the geographic routing. Several techniques such as a mutual witness algorithm [2] and a cross-link detection protocol [9] have been proposed for the geographic routing problems due to the location error. However, these approaches still remain the problems such as the additional cross-links [2] and the high message overheads [9]. Moreover, their approaches do not consider the location accuracy improvement, but solve the geographic routing problems by using the connectivity or probe packets.

In this article, we propose a location estimation and dynamic link detection for the geographic routing in the NLOS environments. In order to compensate the location error due to the NLOS condition, we suggest a location estimation approach, which combines the minimum mean square estimate (MMSE) [10] and the Min-max method [11]. Next, the proposed approach detects the unstable NLOS link through the difference between two Relative Neighborhood Graph (RNG) [12] topologies: one is a RNG topology based on the estimated locations, and the other is a RNG topology based on the ranging distances. With the dynamic link detection approach, the mobile device can adjust to the NLOS link adaptively. Through the proposed approach, each mobile device can obtain its accurate physical location and detect the NLOS wireless link in the geographic routing. Simulation and experimental results show that the proposed approach can enhance the geographic routing performance in the NLOS environment.

The article is organized as follows: in 'Basic overview' section, we present a system overview of the localization and the geographic routing. The NLOS effects in the geographic routing are described in 'Geographic routing problems in NLOS environments' sSection. In 'Proposed approach' section, we propose a location estimation and dynamic link detection approach in the geographic routing. The experimental results and performance evaluations are shown in the 'Performance results' section, and we conclude the article in 'Conclusion' section.

\section{Basic overview}

\section{Localization and ranging techniques}

Localization systems are composed of the two type devices: one is a reference device, which does know its physical location, and the other is a mobile device to estimate its physical location by using the reference devices.
Figure 1 shows the example of the IEEE 802.15.4abased localization system. Before the mobile device estimates its location, the network of the system should be configured. Initially, a base station (BS) searches for any active mobile devices by sending out data packets with destination medium access control (MAC) addresses for devices. If it receives a hardware acknowledgment from the devices with any of those addresses, then it stores the MAC address of the responding device. The MAC addresses of the reference devices are set in the base station, and then they are provided to all mobile devices identified by the base station. Through this procedure, each mobile device can obtain the MAC addresses for all reference devices in the network.

After configuration of the network, each mobile device calculates the distances to the reference devices by exchanging the ranging packets with the neighbor reference devices. Upon collecting of the ranging information, the mobile device estimates its location by using the localization techniques [13]. To obtain the ranging information between the devices, the IEEE 802.15.4a standard utilizes two ranging protocols: TW-TOA and symmetrical double-sided two-way ranging (SDS-TWR) $[4,5,14]$. The TW-TOA is a method to measure the propagation delay of the radio signal between a transmitter and a receiver. According to the IEEE 802.15.4a standard, the ranging frame RFRAME is indicated by setting a ranging bit in the PHY header of the IEEE 802.15.4a packet [4]. As shown in Figure 2a, a ranging distance between two devices is determined typically via two-way exchange of an RFRAME and tracking its arrival time. Consider that a mobile device A wants to calculate the ranging distance to the reference device $\mathrm{B}$. The time of flight of the first arriving signal $T_{\mathrm{p}}$ can be written as:

$$
T_{\mathrm{p}}=\frac{1}{2}\left(T_{\text {round }}-T_{\text {reply }}\right)
$$

where $T_{\text {round }}$ is the time between the departure of a ranging packet from device $A$ and the reception of the reply packet from device $\mathrm{B}$ and $T_{\text {reply }}$ is a turn-around time.

The SDS-TWR is based on the precise time measurements of the signals propagating forward and backwards between two devices [14]. As illustrated in Figure 2b, a signal propagates from one device to another device and again sends back to the original devices.

The time of flight of the signal $T_{\mathrm{p}}$ can be obtained by using the following equations:

$$
T_{\mathrm{p}}=\frac{1}{4}\left(T_{\text {roundA }}-T_{\text {replyA }}+T_{\text {roundB }}-T_{\text {replyB }}\right)
$$

where $T_{\text {roundA }}$ and $T_{\text {roundB }}$ represent the turn-around time at device $\mathrm{A}$ and device $\mathrm{B}$, respectively, and $T_{\text {replyA }}$ 


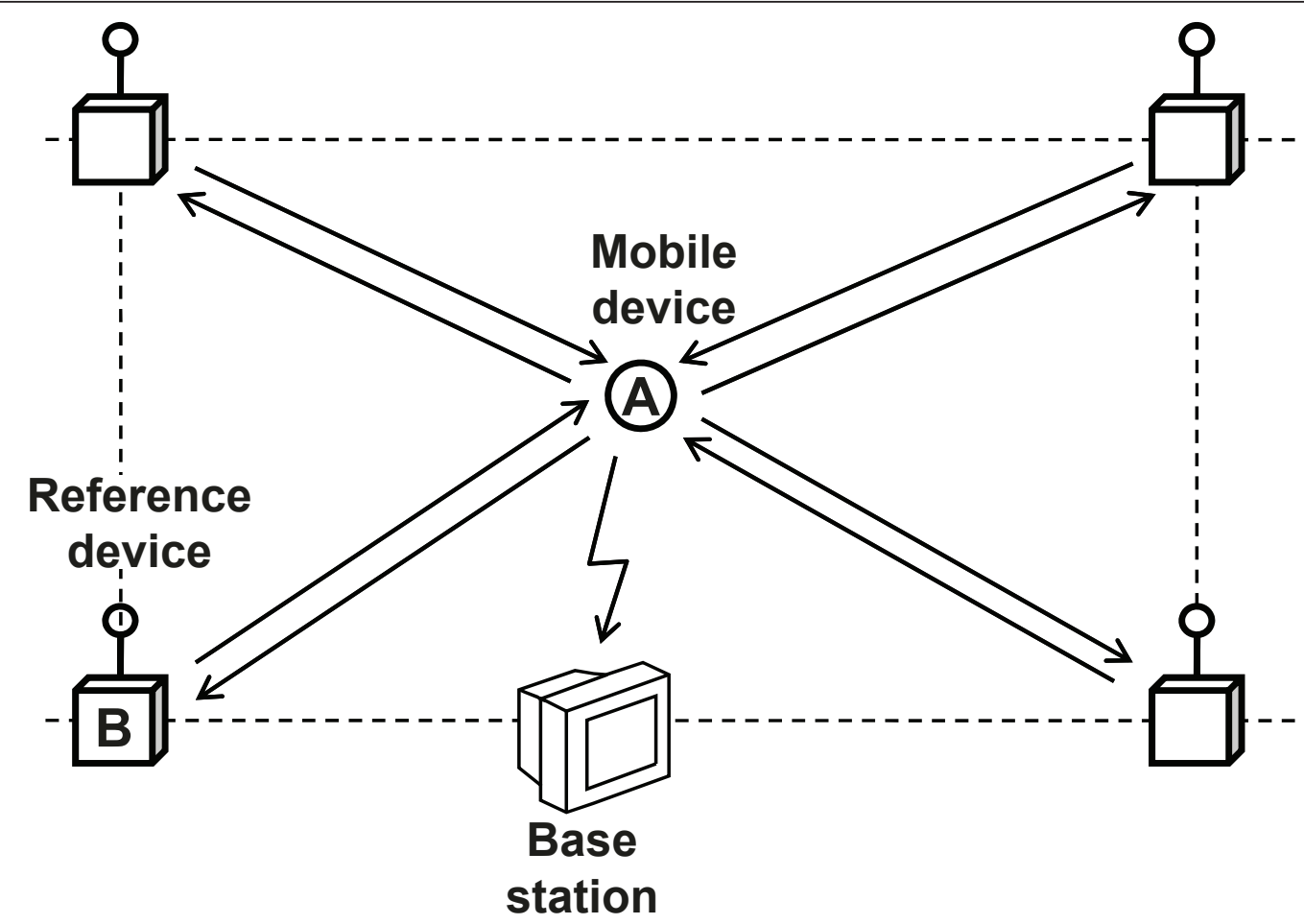

Figure 1 IEEE802.15.4a-based localization system.

and $T_{\text {replyB }}$ are processing delay. Compared with the TW-TOA, the SDS-TWR can eliminate the effects of the clock synchronization and the crystal offset [14].

\section{Geographic routing protocol}

Geographic routing protocol forwards data packets using location information of wireless devices [1]. Compared with other neighbor-based routing protocols such as Dynamic Source Routing (DSR) [15] and Ad-Hoc On-Demand Distance Vector Routing (AODV) [16], the geographic routing can reduce the communication overhead during route search procedure $[1,2]$. The basic operation of the geographic routing protocol is a greedy forwarding, which is a method to select the neighbor device geographically closest to the destination device as next hop [1]. Figure 3a shows the example of the greedy forwarding in the geographic routing protocol. When a device $s$ wants to deliver packets to the destination device $d$, the device $s$ marks the packets with its destination's locations and searches the next hop. If a device knows its neighbors' locations, the locally optimal choice of next hop is the neighbor geographically closest to the packet's destination. In Figure 3a, the device $s$ selects the device $u$ as next hop, and sends the packets to the device $u$. Next, the device $u$ selects the device $v$ as next hop and sends the packets to the device $v$. This forwarding procedure repeats until the packet arrives at the destination device $d$.
When there is a void that a device has no neighbor closest to the destination device, the face routing is used to route the packets around the void [1]. For the face routing, planarization algorithms such as the RNG [12] and the Gabriel graph (GG) [17], which make a network with no cross links, are needed. The geographic routing procedure, which combines greedy forwarding with face routing, is as follows: initially, all data packets are marked as greedy mode. Upon receiving a greedy-mode packet for forwarding, a device searches a device geographically closest to the packets destination. When no neighbor is closer, the device marks the packet into perimeter mode, and performs the face routing. As shown in Figure $3 \mathrm{~b}$, a device $u$ receives packets from a device $s$, the device $u$ searches next hop closest to the destination device $d$. The device $u$ has no neighbor closest to the device $d$, and thus the device $u$ performs the face routing.

\section{Geographic routing problems in NLOS environments}

In this section, we describe the geographic routing problems in the NLOS environments. The IEEE 802.15.4a can provide a framework for low data rate communication with a localization capability. However, when the IEEE 802.15.4a is adapted to the geographic routing, it may have some difficulties in the indoor wireless environments. In most cases, the indoor environments are complex and exposed to a variety of obstacles such as 


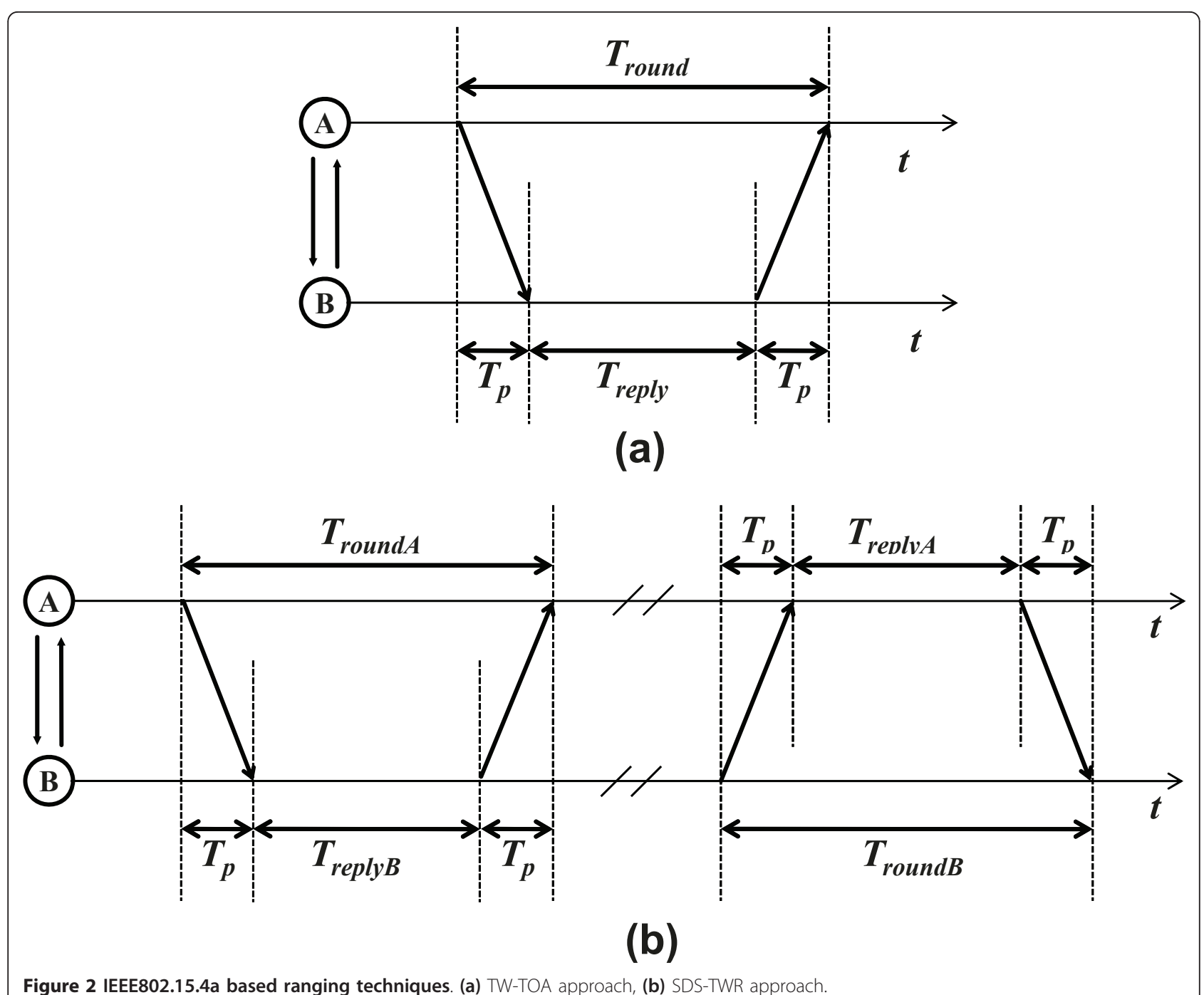

furniture, partitions, and walls. The indoor wireless environments with these obstacles generate the NLOS conditions, which may cause a large scale location error and a highly unstable wireless link in the geographic routing.

The first problem of the NLOS environments is a routing failure due to the large scale location error. When the ranging measurement is corrupted by the NLOS condition, the mobile device has a large scale location error. This inaccurate location information may produce a disconnected link or permanent loop in the geographic routing $[2,9]$.

As shown in Figure 4, there is a NLOS condition between the reference device 2 and the mobile device $s$. When the device $s$ receives the ranging frame from the reference device 2 , the ranging distance is very inaccurate due to the NLOS condition, and its estimated location has a large scale location error. When the device $u$ wants to send data packets to the device $d$, the device $u$ has no neighbors closest to the destination device $d$. Hence, the device $u$ cannot perform greedy forwarding, and it uses face routing to forward the packets. Since the device $s$ has a large scale location error, it removes the edge $(u, w)$ in the planarization procedure. Removal of the edge $(u, w)$ produces a planar graph to be disconnected, and the face routing fails to deliver the packets to the destination device $d$. Therefore, the large scale location error due to the NLOS condition may produce the routing failure in the geographic routing procedure.

The second problem is an unstable wireless link caused by the NLOS condition. When there are any obstacles between the devices, the wireless link is highly unstable due to the NLOS condition. The NLOS link can degrade the performance of the geographic routing protocol. Figure 5 shows the example of the NLOS link in the geographic routing protocol. In Figure 5, when a 


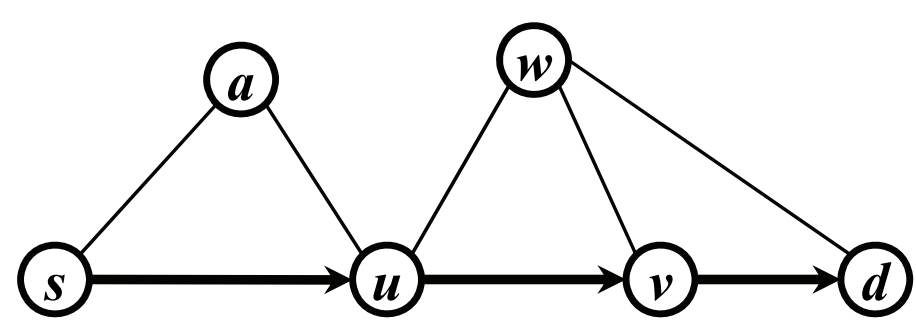

(a)

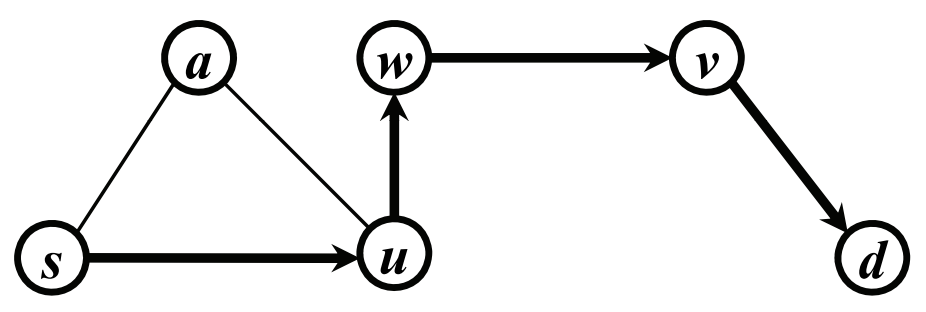

(b)

Figure 3 Geographic routing protocol. (a) Greedy forwarding, (b) face routing.

device $u$ receives the packets from a device $s$, it selects the device $v$ as next hop because the device $v$ is closest to the device $d$. Since the edge $(u, v)$ is an unstable NLOS link, the routing procedure with the edge $(u, v)$ may fail to transmit the packets more frequently than in the line-of-sight (LOS) condition. Therefore, the geographic routing procedure through the NLOS link may cause significant performance degradation in the IEEE 802.15.4a networks.

To analyze the NLOS effects in the IEEE 802.15.4a networks, we conducted the experiments in the indoor environments by using the nanoLOC TRX transceiver

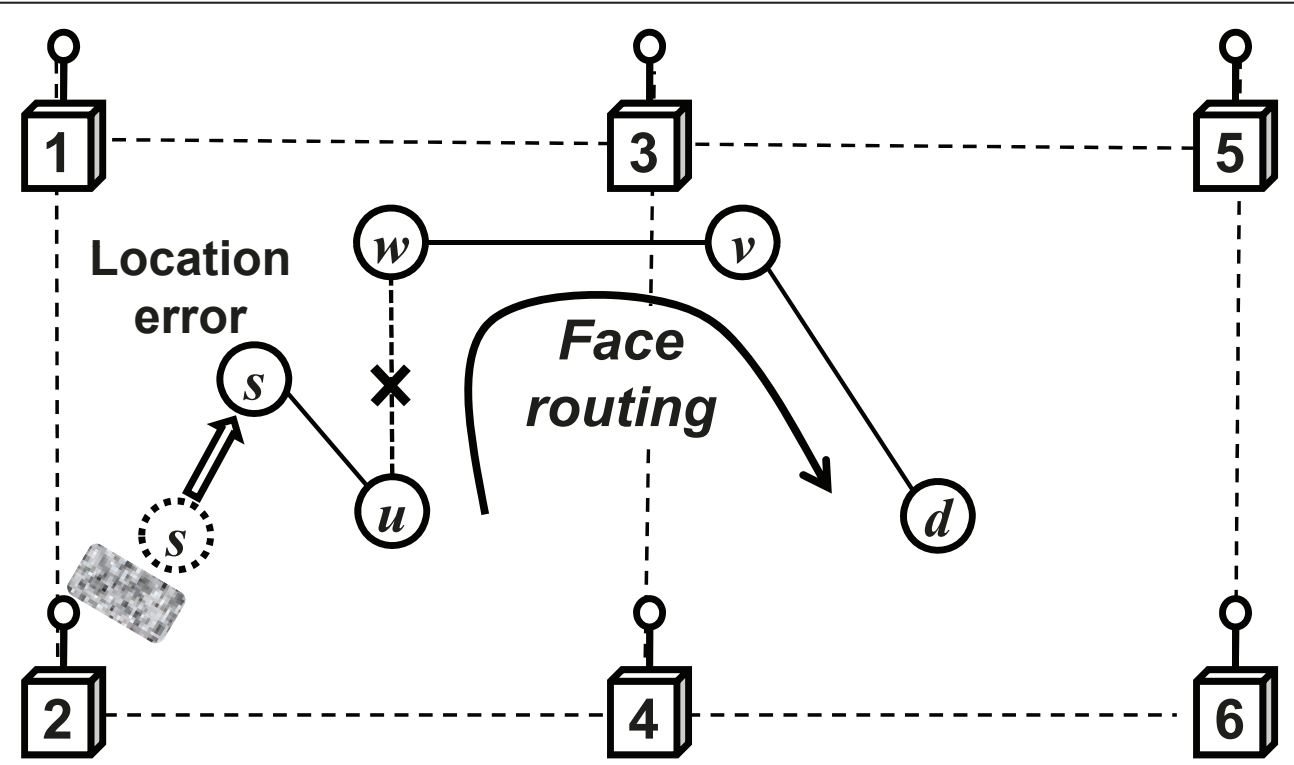

Figure 4 Geographic routing problems of location error in NLOS environments. 


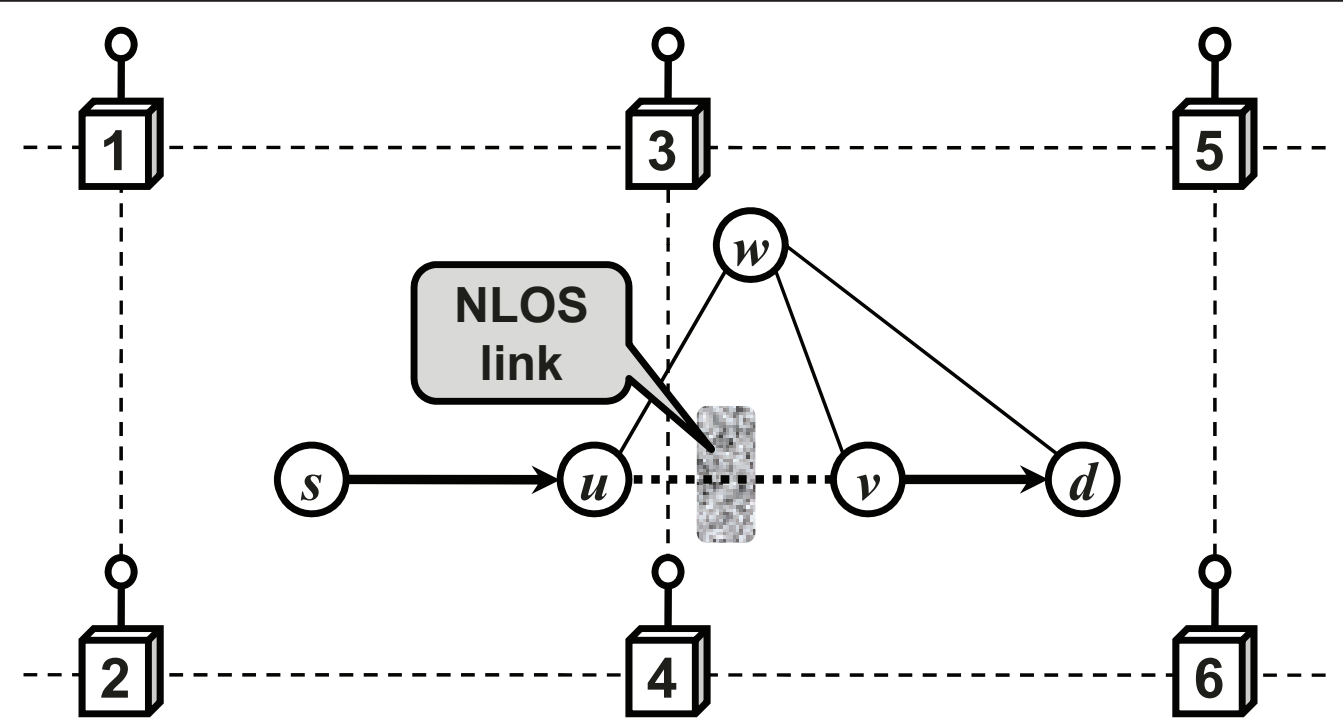

Figure 5 Geographic routing problems of unstable NLOS link.

(NA5TR1) [18], which is compatible with the IEEE 802.15.4a and has a ranging capability based on the SDS-TWR [19]. To analyze the propagation effects on the geographic routing, we measured the packet success rate (PSR) in the indoor environments. In the experiments, we set a device to transmit 100 packets every second, while the other device is listening and counting the number of the received packets. To prevent the degradation from any contention problems due to other devices, we used only two devices for experiments. PSR is measured as the distance between two devices changes, and there is no obstacle between them (i.e., devices are in LOS condition). We measured PSR in LOS condition at the different places: (1) hall without any walls (place A), (2) indoor office at building (place $B$ ), and (3) open space (place C).

Figure 6 shows the experimental PSR in LOS as the distance increases. The experimental results show the different patterns as the place changes. As shown in Figure 6 , for place A, PSR in LOS is almost above $90 \%$. Meanwhile, for place $C$, PSR rapidly decreases as the distance changes. Compared with PSR of place $A$ and place $B$, PSR of place $C$ has more significant packet loss rate. The purpose of our proposed approach is to enhance the geographic routing performance in the complex indoor environments with various obstacles. Therefore, we analyzed more detailed NLOS effects for place $B$.

The ranging measurement results in the indoor environment are shown in Figure 7. The ranging distances are measured under both LOS and NLOS conditions. In order to generate the NLOS condition, we place an absorbing wall between two devices. In Figure 7, the real distance between two devices is about $9.4 \mathrm{~m}$. As shown in Figure 7, under the LOS condition, the ranging distance is close to the real distance and relatively constant over the time. Meanwhile, under the NLOS condition, the ranging distance is very inaccurate and it has large fluctuation over the time. The inaccurate and irregular ranging distance in NLOS condition can produce a large scale location error.

Next, PSR in LOS and NLOS conditions are shown in Table 1. Experimental results show the different patterns in LOS and NLOS conditions. As shown in Table 1, PSR of the NLOS condition is much lower than that of the LOS condition. The experimental results show that the NLOS effects may cause the performance degradation such as the large scale location error and the highly unstable wireless link in the IEEE 802.15.4a-based geographic routing.

\section{Proposed approach}

To improve the geographic routing performance in the NLOS environments, we propose a novel approach that combines location estimation and dynamic link detection. During the geographic routing procedure, the proposed approach estimates the current location of the mobile device and detects the wireless link condition dynamically in the NLOS environments. Through the proposed approach, each mobile device can obtain its accurate physical location and detect the NLOS wireless link in the geographic routing.

\section{Location estimation procedure}

In this procedure, each mobile device estimates its current location through the combination of MMSE and 


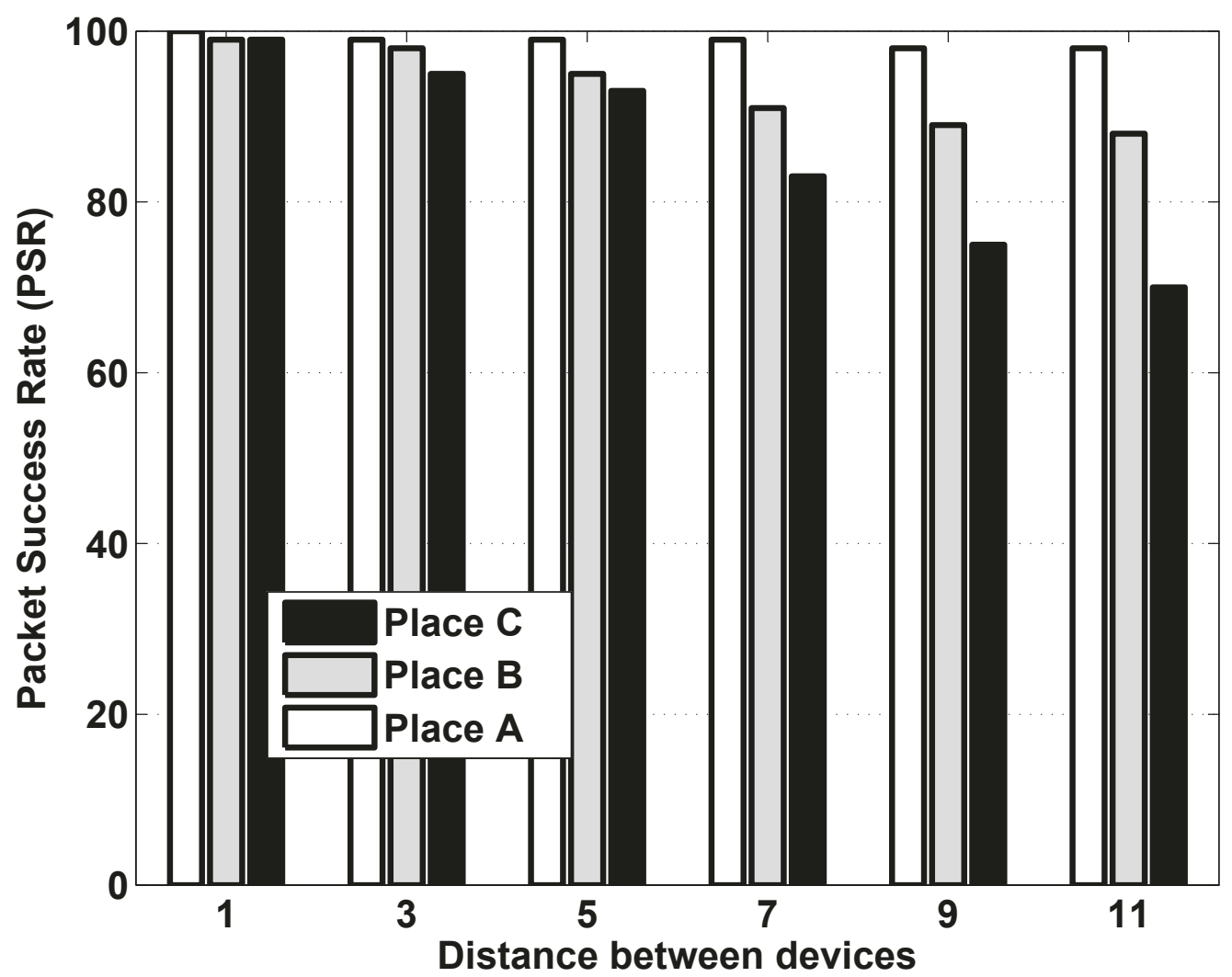

Figure 6 Experimental PSR results as the distance between devices

the Min-max algorithm. In the IEEE 802.15.4a networks, the mobile device exchanges the ranging frames with its neighbor reference devices periodically, and it calculates the ranging distance to the reference device. Upon collecting the ranging distances, the mobile device estimates its physical location. Generally, the location estimation problem of a mobile device $u$ at time $k$ can be formulated using a maximum likelihood estimate (MLE) approach as:

$$
\hat{x}_{u}(k)=\underset{x \in X}{\arg \min } \sum_{j=1}^{m}\left(\left\|x-x_{j}(k)\right\|_{2}-\tilde{d}(u, j)\right)^{2}
$$

where $x_{j}(k)$ is a location of the reference device $j$ at time $k, m$ is a number of neighbor reference devices, and $\tilde{d}(u, j)$ is a ranging distance to the reference device $j$ at time $k$, and \|\|$_{2}$ is the Euclidean norm.

The estimated location with the MLE can be obtained by taking the MMSE approach $[10,20,21]$. When all ranging measurements are in LOS conditions, the MMSE approach enables the mobile device to obtain an accurate physical location. However, if there is any NLOS condition among the ranging measurements, then the estimated location of the MMSE has a large scale location error since the MMSE is very sensitive to the
NLOS error. To compensate the location error due to the NLOS effects, the proposed approach utilizes the Min-max algorithm [11,22]. The Min-max algorithm constructs a bounding box for each reference device by using its position and the ranging distance. The coordinate bounds of the Min-max are given by:

$$
\max \left(x_{j}(k)-\tilde{d}(u, j)\right) \leq x_{\text {Bound }}(k) \leq \min \left(x_{j}(k)+\tilde{d}(u, j)\right)
$$

where $x_{\text {Bound }}(k)$ are the coordinate bounds of the Min-max at time $k$.

Compared with MMSE, the Min-max is less sensitive to the NLOS ranging error since stretching the bounding boxes has little effect on the position of the center [22]. Upon constructing the coordinate bounds, the mobile device checks that its estimated location has a large scale location error. If the ranging distance is corrupted by the NLOS condition between two devices, the estimated location of the MMSE is out of the Min-max boundary, and the proposed approach decides its location as the center of the Min-max boundary. Through the combination of the MMSE and the Min-max, the large scale location error due to the NLOS effects can be effectively compensated.

Figure 8 shows the example of the location estimation procedure of the proposed approach in LOS and NLOS 


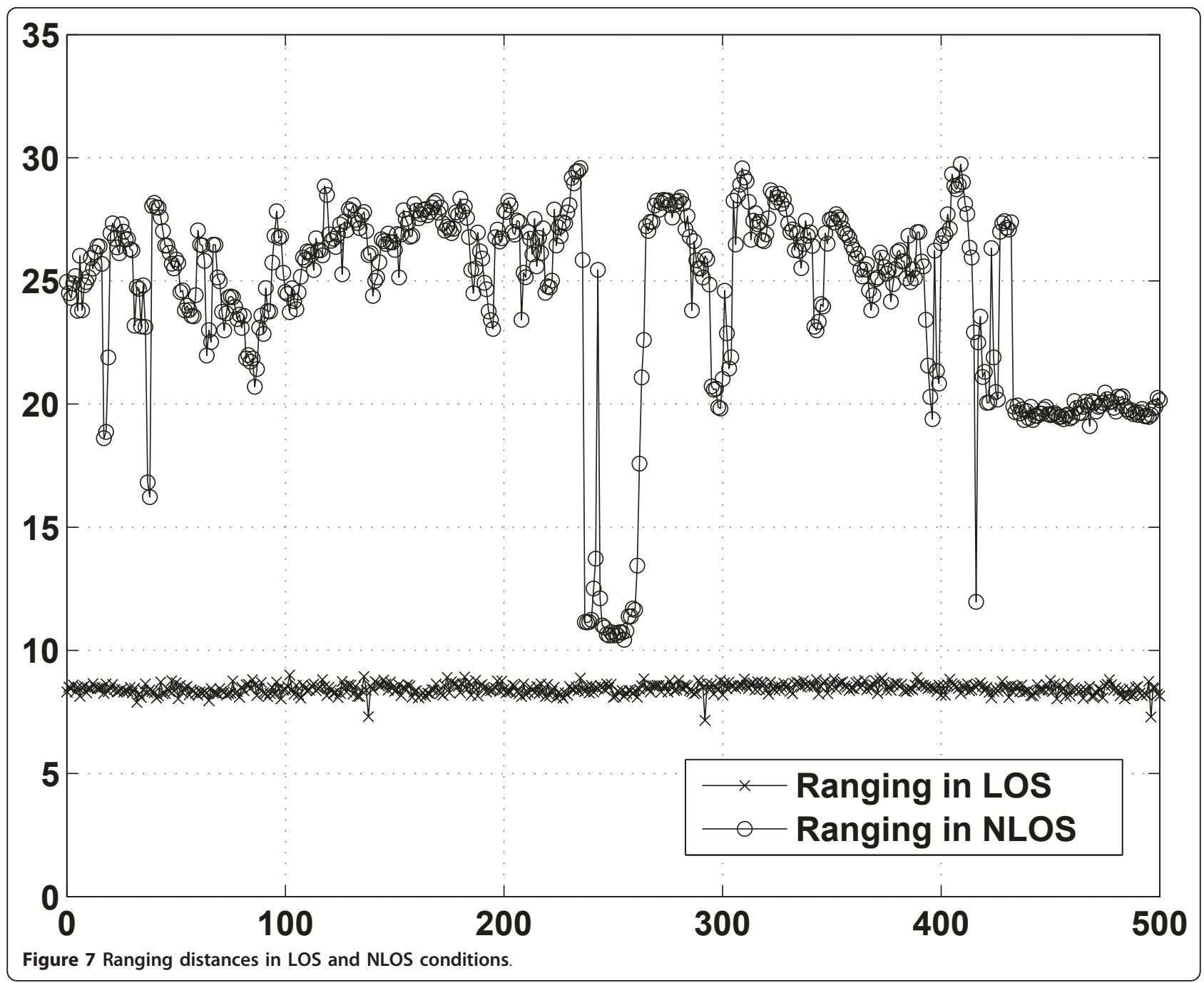

conditions. As shown in Figure 8a, the MMSE of the device $u$ is accurate because the device $u$ is in the LOS environment. Therefore, the estimated location is in the Min-max coordinate bounds and the final location of the device $u$ becomes the estimated location of the MMSE. Meanwhile, as shown in Figure 8b, the MMSE of the device $u$ has a large scale location error since the estimated location of the MMSE is corrupted by the NLOS condition of the reference device $\mathrm{B}$. The estimated location of the MMSE is out of the Min-max coordinate bounds, and the proposed approach decides its location as the center of the Min-max boundary.

Table 1 Packet success rate (\%).

\begin{tabular}{llllll}
\hline Conditions & \multicolumn{6}{l}{ Experiment number } & & \\
& E1 & E2 & E3 & E4 & E5 \\
\hline PSR in LOS & 89.3 & 92.2 & 88.3 & 89.5 & 89.7 \\
PSR in NLOS & 54.2 & 51.4 & 60.9 & 39.1 & 39.1 \\
\hline
\end{tabular}

Through the proposed approach, the mobile device can obtain an accurate physical location in the LOS conditions and compensate the large scale location error in the NLOS conditions.

\section{NLOS link detection procedure}

Although the above location estimation procedure can compensate the large scale location error, the unstable wireless link caused by the NLOS condition may still disturb the geographic routing procedure. Moreover, it is difficult for the mobile device to detect the NLOS link accurately, since the LOS and NLOS conditions of the mobile device are continuously changing. To solve the NLOS link problems, we propose a dynamic link detection approach for the NLOS environments. In the geographic routing, each mobile device periodically performs the RNG or GG planarization by using the beacon messages received from its neighbor devices. Based on the information of the received beacon messages, the 


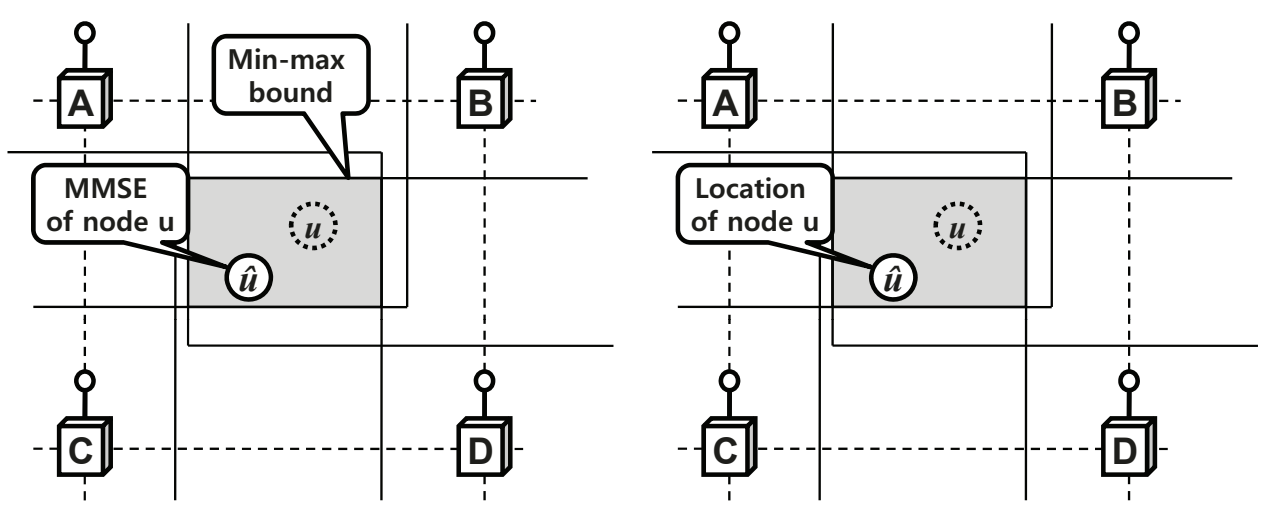

(a)
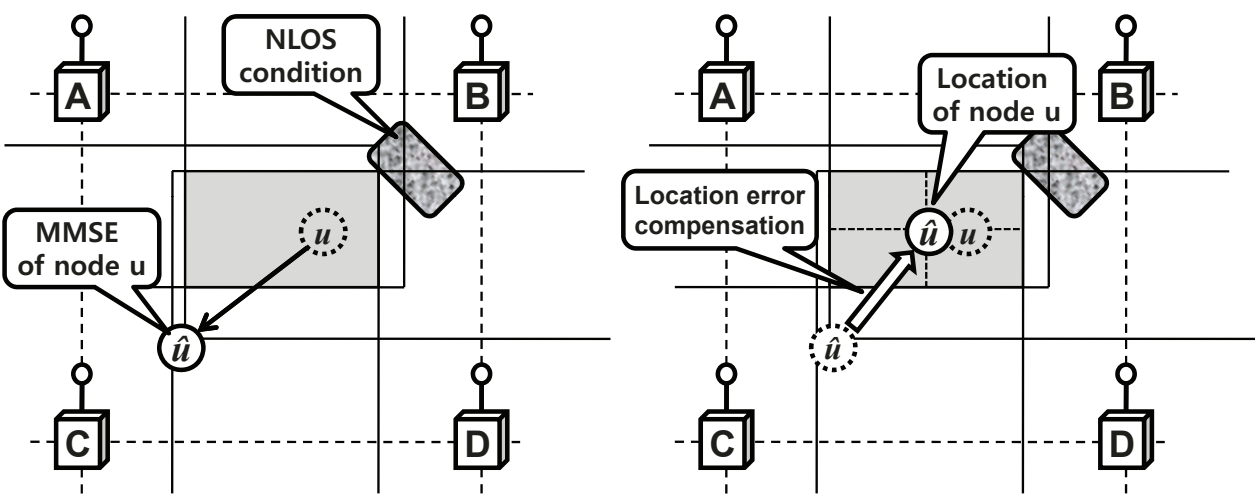

(b)

Figure 8 Location error compensation procedure. (a) Location estimation in LOS condition, (b) location estimation in NLOS condition.

proposed approach constructs the two RNG topologies: One is an RNG topology based on the location through the proposed location estimation, and the other is an RNG topology based on the ranging distances with the neighbor devices.

Generally, RNG is defined as follows: an edge $(u, v)$ exists between devices $u$ and $v$ if the distance $d(u, v)$ is less than or equal to the distance between every other device $w$, and whichever of $u$ and $v$ is farther from $w$. The statement can be written in equational form:

$$
\forall w \neq u, v: d(u, v) \leq \max [d(u, w), d(v, w)]
$$

where $d(u, v)$ is a distance between the device $u$ and the device $v$.

Figure 9 shows the example of RNG topology. In Figure 9, the edge $(u, v)$ is not included in RNG graph, since there exist a device $w$ in the intersection of two circles centered at $u$ and $v$ and with radius $d(u, v)$.

For device $u$, the RNG topology based on the estimated location is constructed as follows:

$$
\forall w \neq u, v: \hat{d}(u, v) \leq \max [\hat{d}(u, w), \hat{d}(v, w)]
$$

where $\hat{d}(u, v)$ is the distance calculated from the location information of two devices $u$ and $v$.

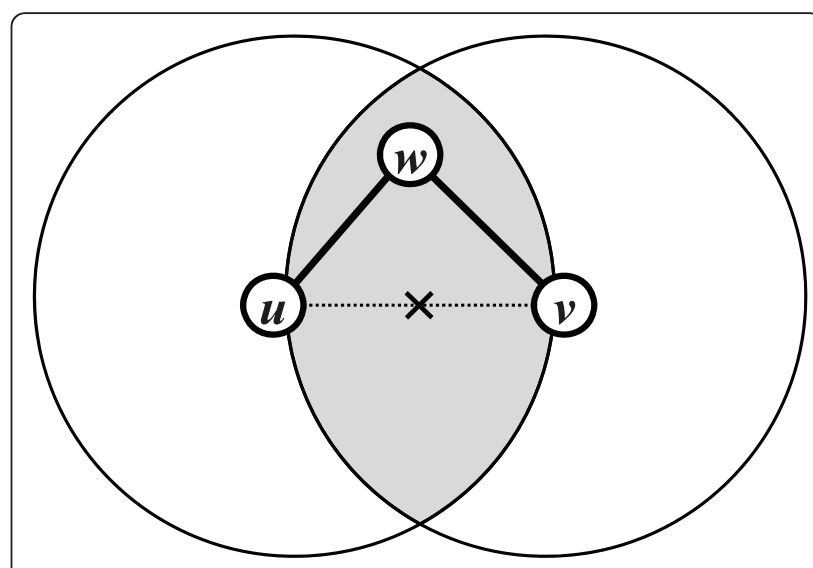

Figure 9 Example of RNG. The edge $(u, v)$ is not included in RNG graph because of device $w$. 
Although devices do not know their own locations, they can achieve RNG topology construction if they have capability to calculate the ranging distances [23]. In the ranging-based RNG topology, each device broadcasts its periodic message including the list of its neighbors with ranging distances. Upon receiving the message, the device $u$ constructs the ranging based RNG topology. An edge $(u, v)$ exists between two devices $u$ and $v$ if its ranging distance is less than or equal to the ranging distance between every other device $w$. The statement can be written in equational form:

$$
\forall w \neq u, v: \tilde{d}(u, v) \leq \max [\tilde{d}(u, w), \tilde{d}(v, w)]
$$

where $\tilde{d}(u, v)$ is the ranging distance between two devices $u$ and $v$.

After construction of two RNG topologies, the device $u$ checks that two RNG topologies are different. As shown in Figure 10, in the location based RNG topology, the device $u$ is connected to the device $v$. Meanwhile, in the ranging distance based RNG topology, the device $u$ is disconnected to the device $\mathrm{v}$ because the ranging distance $\tilde{d}(u, v)$ is corrupted by the NLOS condition. By comparing these two RNG topologies, the device $u$ detects the NLOS link $(u, v)$. By using the difference between two RNG topologies, the proposed approach can detects the wireless NLOS link condition adaptively.

If the NLOS range estimate between the mobile device and the reference device is detected through the difference between two RNG topologies, the mobile device improves its location accuracy through the location refinement procedure. In this procedure, the mobile device recalculates its location with the remaining LOS range estimates. Figure 11 shows the example of the location refinement procedure in the NLOS environment.

First, as shown in Figure 11a, the mobile device $u$ constructs two RNG topologies. In the location-based RNG topology, the device $u$ is connected to the device $\mathrm{v}$. Meanwhile, in the ranging distance based RNG topology, edge $(u, v)$ is disconnected since the NLOS ranging estimate $\tilde{d}(u, v)$ is larger than the distance $\tilde{d}(u, w)$ and the distance $\tilde{d}(v, w)$. By using the difference of these two RNG topologies, the device $u$ can detect the NLOS link $(u, v)$. Next, as shown in Figure 11b, the device $u$ recalculates its location by using the MMSE with LOS range estimates $\tilde{d}(u, w), \tilde{d}(u, x)$, and $\tilde{d}(u, y)$. The estimated location $\hat{x}_{u}(k)$ can be written as:

$$
\hat{x}_{u}(k)=\underset{x \in X}{\arg \min } \sum_{(u, j) \in \operatorname{LOS}}\left(\left\|x-x_{j}(k)\right\|_{2}-\tilde{d}(u, j)\right)^{2}
$$

where $x_{j}(k)$ is a location of the reference device $j$ at time $k,(u, j) \in$ LOS indicates that edge $(u, j)$ is a LOS link, and $\tilde{d}(u, j)$ is a LOS range estimate to the reference device $j$ at time $k$.

Through the location refinement procedure, each mobile device can improve its location accuracy in the NLOS environment.

Figure 12 shows the example of the proposed NLOS link detection scheme in the geographic routing. As shown in Figure 12a, there exists the NLOS link between the device $u$ and the device $v$, which is closest to the destination device $d$. In the conventional geographic routing, the mobile device $u$ chooses the next hop as a device $v$, since the device $v$ is closest to the destination device $d$. In the geographic forwarding procedure, the NLOS link $(u, v)$ may degrade the routing performance. Figure 12b shows the proposed NLOS link detection approach in the geographic routing procedure. As shown in Figure 12b, the device $u$ detects the NLOS link $(u, v)$ by comparing these two RNG topologies. Therefore, in the geographic forwarding procedure, it selects next hop as device $w$. The proposed NLOS link detection can enhance the performance of geographic routing in the NLOS environments.

\section{Performance results}

To analyze the performance of the proposed approach, we conducted the experiments by using the CSS-based NA5TR1 [18]. The NA5TR1 operates in the $2.4 \mathrm{GHz}$ license-free ISM band and supports the ranging capability based on the SDS-TWR [19]. Figure 13 shows the device deployment and the experimental network topology. In the experiments, six reference devices are
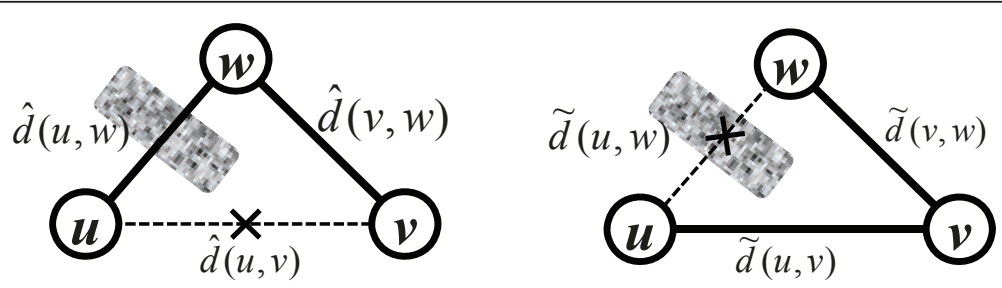

Figure 10 RNG construction. (a) Location-based RNG, (b) ranging distance-based RNG. 


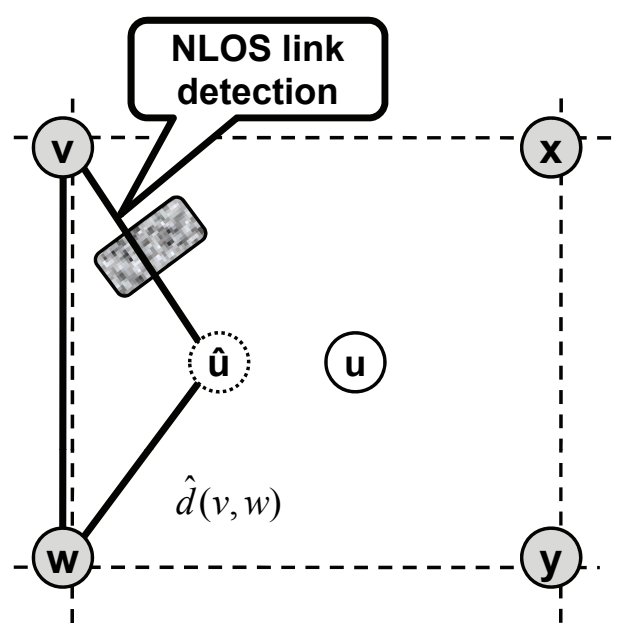

(a)

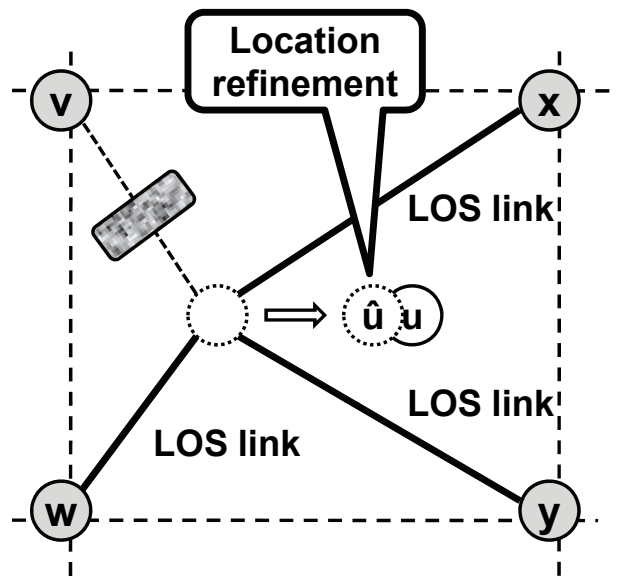

(b)

Figure 11 Location refinement procedure. (a) NLOS link detection, (b) location refinement with LOS range estimates.

deployed in the corner and seven mobile devices are distributed in the indoor places. For the network configuration, a base station device, which is connected to a personal computer $(\mathrm{PC})$, searches for any active devices. If the base station receives an ACK message from any device, then it stores the MAC address of the responding device and provides it to other mobile devices. The ranging distances are calculated in each device by using the SDS-TWR, and the base station collects the ranging information and estimates the physical location for each device. The situations of the devices are set as the following scenarios: first, the range estimate of one reference device is in NLOS condition (device $x$ in Figure 13). Second, the range estimates of all reference devices are in LOS conditions (the other devices in Figure 13). To analyze the location accuracy of the NLOS effects, we place the obstacle between the device $x$ and the reference device.
First, we compared the performance of five localization algorithms in terms of the location error: (1) MMSE, (2) residual weighting algorithm (Rwgh), (3) localization scheme in [24] (LPMD-1), (4) localization scheme in [25] (LPMD-2), and (5) the proposed approach (Proposed). Rwgh was proposed in [26] to mitigate the NLOS errors in the TW-TOA-based localization. The localization procedure of Rwgh is as follows: first, Rwgh forms the combinations of the $m$ range measurements and computes the intermediate MMSE for each combination. Next, Rwgh finds the location as the weighted linear combination of the intermediate MMSE estimates. In [24], the authors proposed a novel NLOS localization scheme with time of arrival (TOA) and angle of arrival (AOA) measurement data. The LPMD-1 in [24] is composed of the following two steps: first step is to determine the centroid among the cluster of Line of Possible Mobile Device Location (LPMD), which

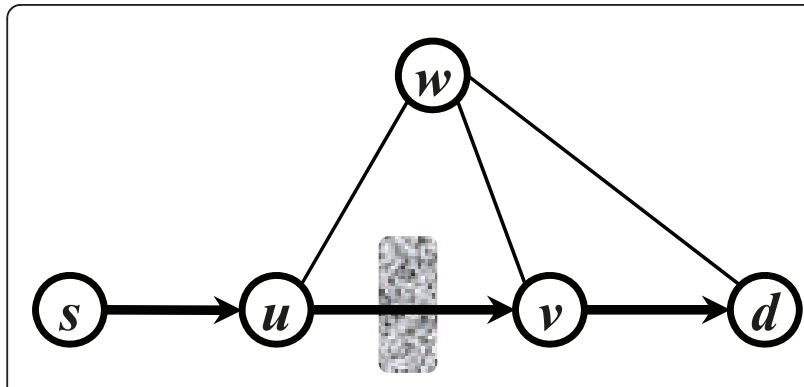

(a)

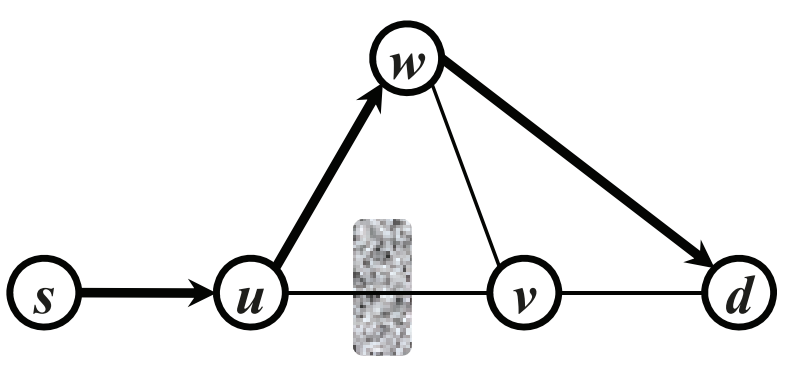

(b)

Figure 12 Geographic routing in NLOS environments. (a) Conventional geographic routing, (b) geographic routing with the proposed link detection. 


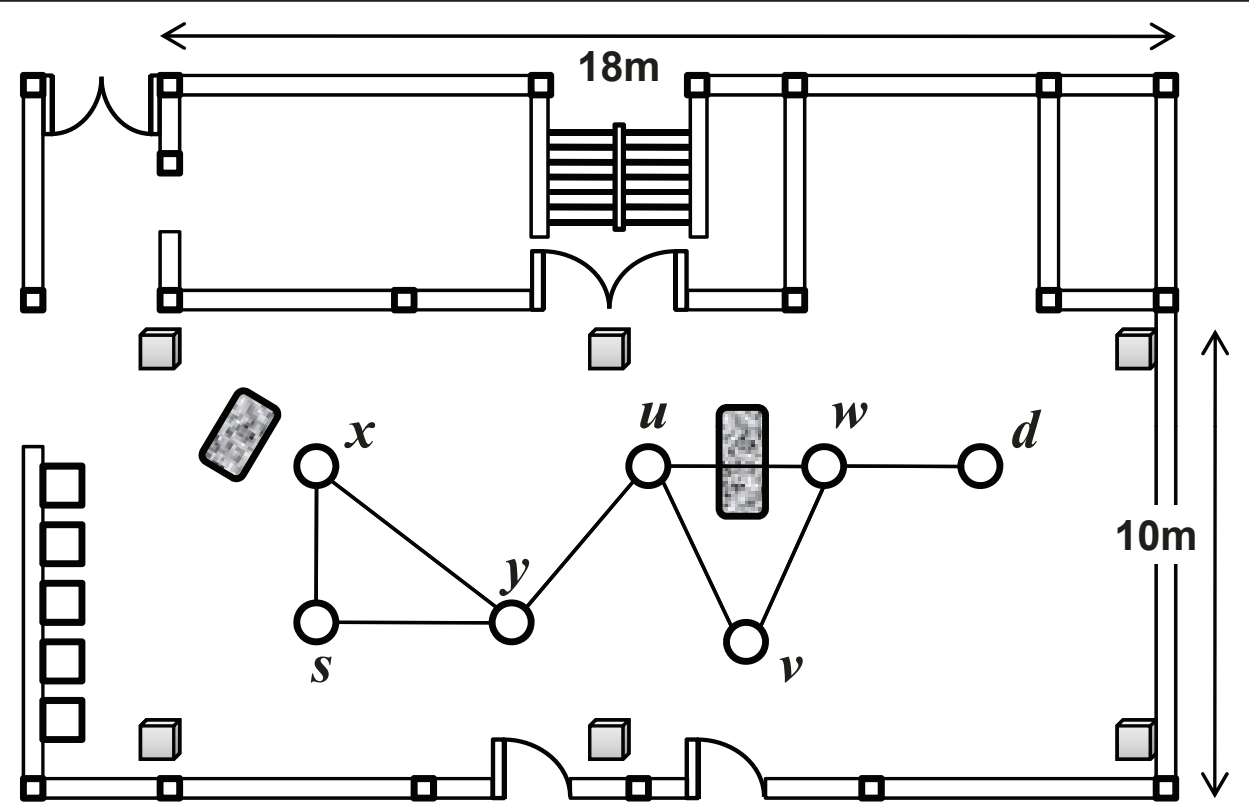

Figure 13 Device deployment and the experimental network topology.

derives from the measured TOA and AOA at the reference devices. These LPMDs are the lines that containing the possible mobile device location. Second step is to find the appropriate pair of LPMDs that has the shortest Euclidean distance from the centroid and choose it as the mobile device location. In [25], the proposed scheme (LPMD-2) derives the LPMDs from the TOA and AOA measurements at the reference nodes. Next, the proposed scheme estimates the mobile device locations by using the weighted average of the each signal paths.

Table 2 shows the experimental location error in terms of each algorithm. As shown in Table 2, the location error in LOS condition is smaller than the location error in NLOS condition (device $x$ ). The location error in NLOS condition (device $x$ ) shows the different results for each localization algorithm. In MMSE and Rwgh, the device $x$ has a large scale location error, since their approaches do not compensate the location error due to the NLOS effects. Meanwhile, the location errors of LPMD-1 and LPMD-2 are smaller than those of MMSE and Rwgh. The reason is that the centroid $C$ with the LPMDs separates the LPMDs of the multiple bound

Table 2 Localization accuracy

\begin{tabular}{lll}
\hline Algorithms & $\begin{array}{l}\text { Location error in LOS } \\
\text { (Other devices) }(\mathbf{m})\end{array}$ & $\begin{array}{l}\text { Location error in NLOS } \\
(\mathbf{d e v i c e} \boldsymbol{x})(\mathbf{m})\end{array}$ \\
\hline MMSE & 2.3 & 5.3 \\
Rwgh & 2.3 & 5.6 \\
LPMD-1 & 3.0 & 3.8 \\
LPMD-2 & 1.7 & 2.1 \\
Proposed & 2.3 & 3.0 \\
\hline
\end{tabular}

scattering NLOS paths [24]. The location error of the proposed approach is also smaller than those of MMSE and Rwgh, since the proposed approach compensates the large scale location error due to the NLOS effect through the combination of MMSE and Min-max algorithms.

Next, we analyzed the performance of the geographic routing in the NLOS environments. The geographic routing of the proposed approach is performed based on the estimated locations. In Figure 13, the source device $s$ sends the data packets to the destination device $d$ every second. To analyze the effects of the NLOS link, we placed the obstacle between the device $u$ and the device $w$, and measured the PSR and the average path length (APL). In Table 3, conventional approach means the conventional geographic routing without NLOS detection. As shown in Table 3, APL of the proposed approach is larger than APL of the conventional approach, and PSR of the proposed approach is higher than that of the conventional approach. The reason is that the conventional approach does not efficiently mitigate the NLOS effects of the link $(u, w)$ in the geographic routing procedure. Meanwhile, in the proposed approach, the device $u$ detects the NLOS link $(u, w)$ by using the proposed approach and selects next hop as device $v$ in the geographic routing procedure.

Table 3 Geographic routing performance

\begin{tabular}{lll}
\hline Performance results & Conventional & Proposed \\
\hline APL & 5.0 & 6.0 \\
PSR (\%) & 38.1 & 51.1 \\
\hline
\end{tabular}


Experimental results show that the proposed approach can enhance the network performance of the geographic routing protocol in the NLOS environments.

To further analyze the effects of the NLOS environments, we expanded the evaluation of the proposed approach through the large-scale simulations. All simulation parameters are based on the experimental results. The measurement model is composed of the LOS and the NLOS models. In the LOS condition $\left(H_{0}\right)$, the ranging estimate between the device $i$ and the device $j$ is modeled as unbiased Gaussian estimates [21] and it can be written as:

$$
H_{0}: \tilde{d}_{i j}(k)=d_{i j}(k)+n_{i j}
$$

where $H_{0}$ is the LOS condition at time step $k, \tilde{d}_{i j}(k)$ is the LOS ranging distance, $d_{i j}(k)$ is the real distance, $n_{i j}$ is Gaussian random variable of zero-mean with standard deviation.

In the NLOS condition $\left(H_{1}\right)$, the ranging estimate between $i$ and $j$ is modeled as positively biased Gaussian estimate [27] and it can be written as:

$$
H_{1}: \tilde{d}_{i j}(k)=d_{i j}(k)+n_{i j}+e_{i j}
$$

where $H_{1}$ is the NLOS condition at time step $k, \tilde{d}_{i j}(k)$ is the NLOS ranging distance, $d_{i j}(k)$ is the real distance, $n_{i j}$ is Gaussian random variable of zero-mean with standard deviation, and $e_{i j}$ is the NLOS positive bias error.

The measurement noise and the NLOS bias error are obtained through the experimental results conducted in the indoor fields. To obtain the mean square error (MSE) results for the location error, the 100 independent simulation runs are used to evaluate the localization performance. The MSE for the location error $\left(\sigma_{u}\right)$ is defined as follows:

$$
\sigma_{u}{ }^{2}=\sum_{i=1}^{N} \frac{\left(x_{u}-\hat{x}_{u}\right)^{2}+\left(y_{u}-\hat{y}_{u}\right)^{2}}{N}
$$

where $N$ is the number of the simulation runs, $\left(x_{u}, y_{u}\right)$ is the true location of device $p$, and $\left(\hat{x}_{u}, \hat{y}_{u}\right)$ is the estimated location of device $u$.

Table 4 shows the simulation parameters for the simulation.

We analyzed the location accuracy of five localization algorithms in the simulation. Figure 14 and Table 5 shows the average location error and estimated location in the simulation respectively. As shown in Figure 14a, 25 reference devices are deployed in a grid manner and 16 mobile devices are distributed uniformly in a square region. Next, we randomly select some links between the reference device and the mobile device, and set the
Table 4 Simulation parameters

\begin{tabular}{ll}
\hline Simulation parameters & Value \\
\hline Network diameter & $100 \mathrm{~m} \times 100 \mathrm{~m}$ \\
Number of reference devices & 25 \\
Number of mobile devices & 16 \\
Transmit range & $30 \mathrm{~m}$ \\
Measurement noise of ranging distance & $3 \mathrm{~m}$ \\
NLOS bias error of ranging distance & $10 \mathrm{~m}$ \\
\hline
\end{tabular}

selected links to the NLOS link. Estimated locations in terms of each algorithm are plotted in Figure 14b to $\mathrm{f}$. As shown in Figure 14b, c, when there is no NLOS range estimate, the estimated location is close to the true location. Meanwhile, when the range estimate includes the NLOS link, the location error of MMSE and Rwgh is large because their approaches do not reduce the impact of NLOS ranging error. As shown in Figure 14d-to e, the location errors of LPMD-1, LPMD2 , is lower than those of Rwgh and MMSE. The proposed approach compensates the location error due to the NLOS condition through the combination of the MMSE and Min-max approaches. Simulation results show that our proposed approach efficiently reduces the localization error in the NLOS environment.

Based on the estimated locations for each algorithm, we performed the geographic routing simulations. In the simulations, a source device and a destination device are chosen in the network, and the source device sends the data packets to the destination device. To construct the NLOS conditions, we randomly selected the links in the simulation topology and inserted the NLOS effects to the selected links. To analyze the performance of the proposed approach, we compared the proposed approach with conventional approach (conventional). Conventional approach means the conventional geographic routing without any NLOS detection scheme.

First, we analyzed the geographic routing performance as the distance between the source device $(S)$ and the destination device $(D)$ changes. Figure 15 shows the routing performance as the distance between $\mathrm{S}$ and $\mathrm{D}$ changes. As shown in Figure 15, as the distance between $\mathrm{S}$ and D increases, APL of both approaches increases. When the distance is above $60 \mathrm{~m}$, PSR of the proposed approach is higher than that of the conventional approach. The reason is that the proposed approach detects the NLOS link while performing the geographic forwarding procedure.

Next, we analyzed the routing performance as the number of NLOS links changes. In Figure 16a, APL of both approaches increase as the number of NLOS links increases. When the number of NLOS links is more than 3, APL of the proposed approach is higher than that of the conventional approach. The reason is that 

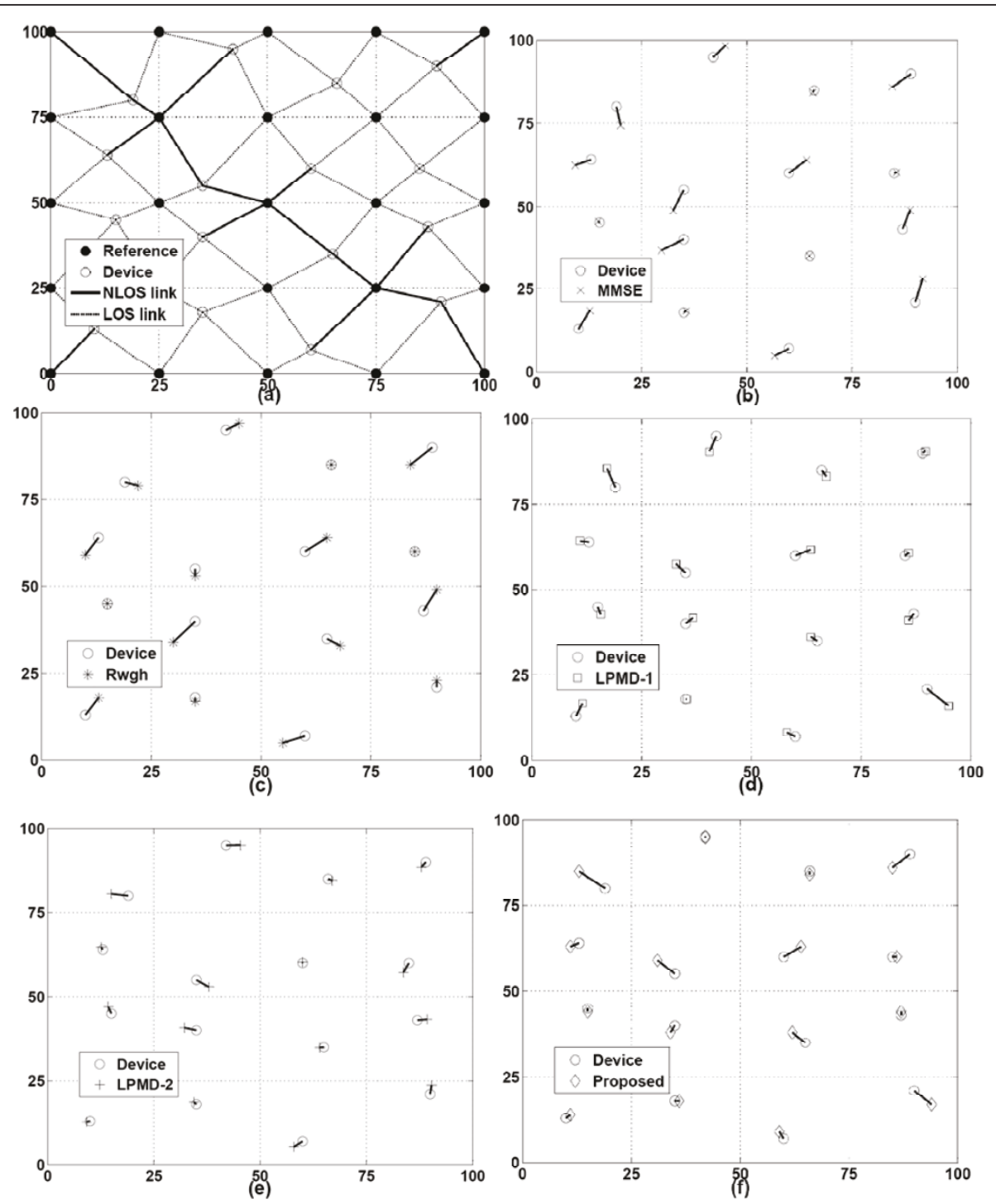

Figure 14 Estimated locations in the NLOS environment. (a) Simulation topology, (b) MMSE, (c) Rwgh, (d) LPMD-1, (e) LPMD-2, (f) proposed.

the proposed approach detects the NLOS link and the link detection scheme increases APL of the proposed approach. In Figure 16b, PSR of both approaches decrease as the number of the NLOS links increases. When the number of NLOS links is more than 3, PSR of the conventional approach rapidly decrease because of the NLOS links. Meanwhile, PSR of the proposed approach is higher than that of the conventional approach, since the proposed approach detects the NLOS link in the geographic routing procedure. Simulation results show that the proposed approach efficiently

Table 5 MSE $\left(\sigma_{u}\right)$ results in simulations

\begin{tabular}{llllll}
\hline Algorithms & MMSE & Rwgh & LPMD-1 & LPMD-2 & Proposed \\
\hline MSE $\left(\sigma_{u}\right)(\mathrm{m})$ & 6.1 & 5.3 & 4.5 & 2.8 & 3.6 \\
\hline
\end{tabular}

mitigates the large location error and improves the geographic routing performance in the NLOS environments.

\section{Conclusions}

The IEEE 802.15.4a-based geographic routing protocol can enhance the network performance in the indoor environments. However, the NLOS environments may cause the performance degradation in the geographic routing protocol. In this article, we propose a location error correction and dynamic link detection for the geographic routing in the indoor NLOS environments. To analyze the performance of the proposed approach, we have conducted the experiments and the simulations. The performance results show that the proposed approach enhances the network performance of the 


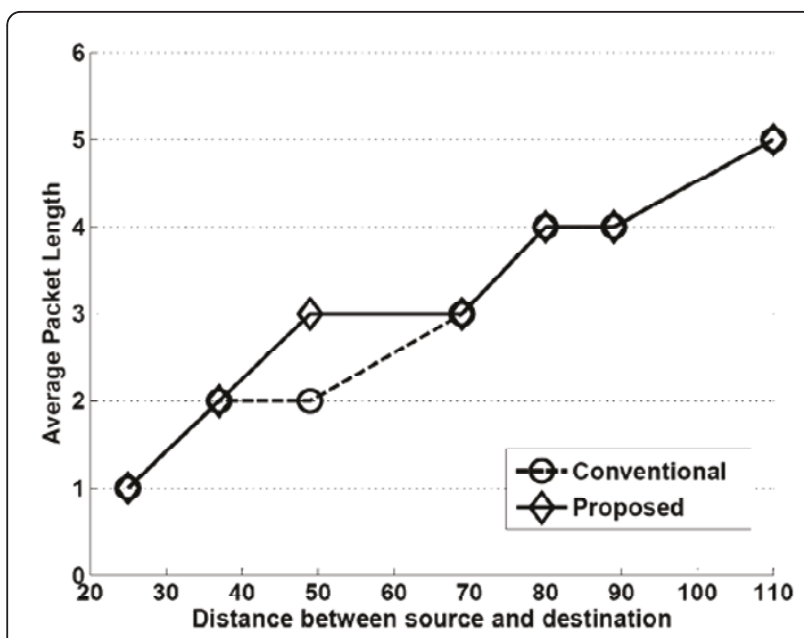

(a) APL vs. distance between S and D

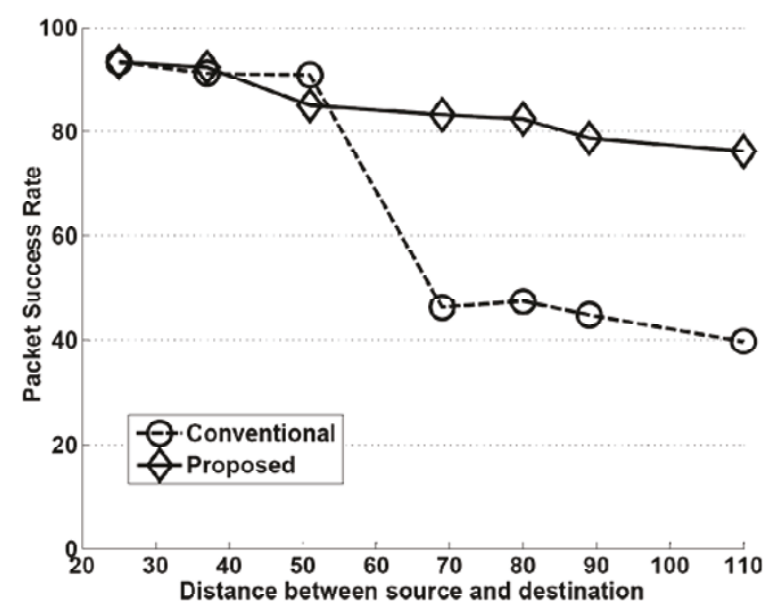

(b) PSR vs. distance between S and D

Figure 15 Routing performance versus the distance between $S$ and D. (a) APL versus distance between $S$ and D and (b) PSR versus distance between $\mathrm{S}$ and $\mathrm{D}$.

IEEE 802.15.4a-based geographic routing in the NLOS environments.

\section{List of abbreviations}

AOA: angle of arrival; AODV: Ad-Hoc On-Demand Distance Vector Routing: APL: average path length; BS: base station; CSS: chirp spread spectrum; DSR: Dynamic Source Routing; GG: Gabriel graph; GPS: global positioning system; LPMD: Line of Possible Mobile Device Location; LR-WPANs: Iow rate wireless personal area networks; MAC: medium access control; MMSE: minimum mean square estimate; MSE: mean square error; NA5TR1: nanoLOC TRX Transceiver; NLOS: non-line-of-sight; PC: personal computer; PHYs: physical layers; PSR: packet success rate; RNG: Relative Neighborhood Graph; SDSTWR: symmetrical double-sided two-way ranging; TOA: time of arrival; TWTOA: two-way time-of-arrival; UWB: ultra-wideband.

\section{Acknowledgements}

This paper was supported by Konkuk University in 2010

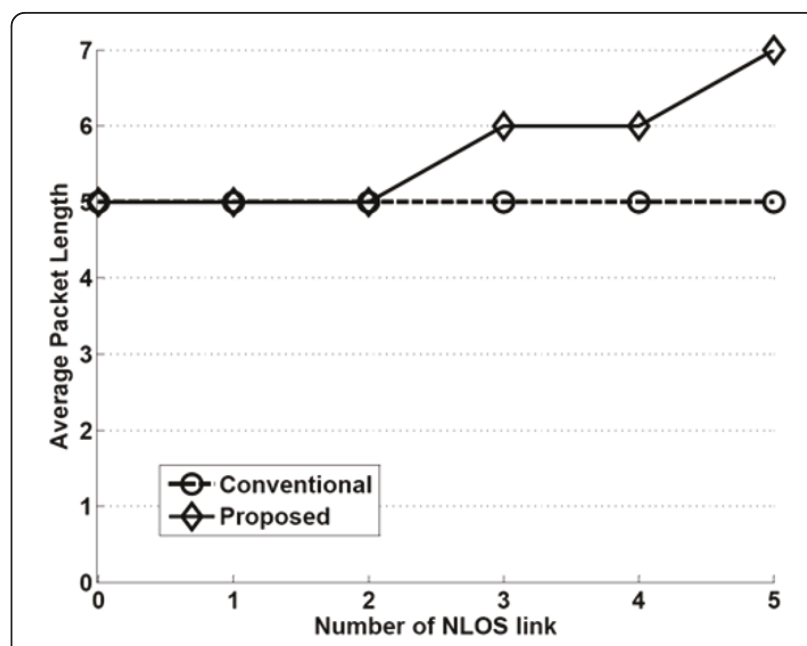

(a) APL vs. the number of NLOS link

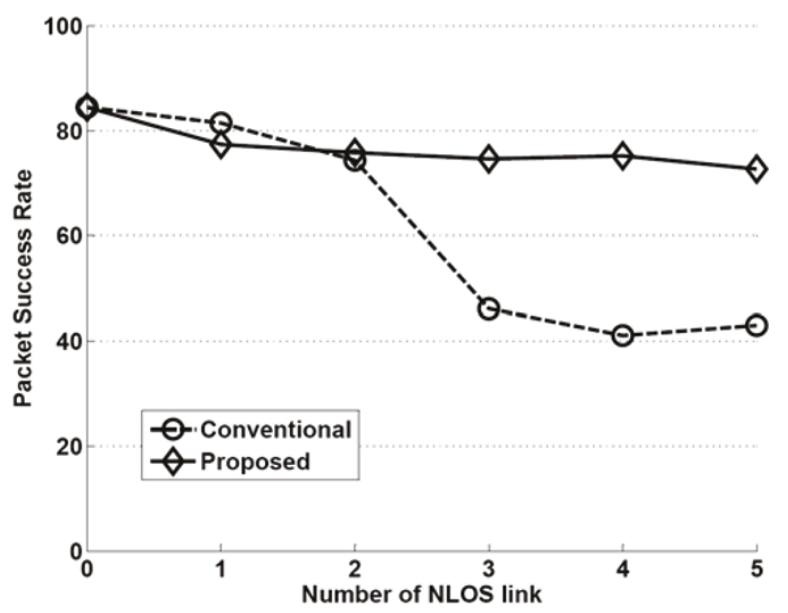

(b) PSR vs. the number of NLOS link

Figure 16 Routing performance versus the number of NLOS link. (a) APL versus the number of NLOS link and (b) PSR versus the number of NLOS link.

Author details

'Department of Electrical Engineering, Konkuk University, Seoul, Korea ${ }^{2}$ Department of Electronic Engineering, Korea University, Seoul, Korea

\section{Competing interests}

The authors declare that they have no competing interests.

Received: 30 November 2010 Accepted: 3 August 2011

Published: 3 August 2011

\section{References}

1. B Karp, HT Kung, GPSR, greedy perimeter stateless routing for wireless networks, in Proceedings of MOBICOM, Boston, USA. 243-254 (Aug 2000)

2. K Seada, A Helmy, R Govindan, On the effect of localization errors on geographic face routing in sensor networks, in Proceedings of IPSN, Berkely, USA. 71-80 (2004)

3. IEEE P802.15.4a/D4 (Amendment of IEEE Std 802.15.4), Part 15.4: Wireless Medium Access Control (MAC) and Physical Layer (PHY) Specifications for Low-Rate Wireless Personal Area Networks (LWR-PANs), (July 2006) 
4. Z Sahinoglu, S Gezici, Ranging in the IEEE 802.15.4a standard. in Proceedings of IEEE WAMICON, Florida, USA, 1-5 (December 2006)

5. MK Oh, JH Park, JY Kim, IR-UWB packet-based precise ranging system for uhome networks. IEEE Trans Consum Electron. 55(1), 119-125 (2009)

6. Y Kong, Y Kwon, G Park, Robust localization over obstructed interference for inbuilding wireless applications. IEEE Trans Consum Electron. 55(1), 105-111 (2009)

7. S Cho, Localization of the arbitrary deployed APs for indoor wireless location-based applications. IEEE Trans Cons Electron. 56(1), 105-111 (2009)

8. S Zhou, JK Pollad, Position measurement using Bluetooth. IEEE Trans Consum Electron. 52(2), 555-558 (2006). doi:10.1109/TCE.2006.1649679

9. Y Kim, R Govindan, B Karp, S Shenker, Geographic routing made practical. in Proceedings of NSDI Boston, USA. 217-230 (April 2005)

10. A Savvides, CC Han, MB Strivastava, Dynamic fine-grained localization in adhoc networks of sensors. in Proceedings of 7th International Conference on Mobile Computing and Networking (MOBICOM), 166-179 (2001)

11. A Savvides, HM Park, MB Strivastava, The n-Hop multilateration primitive for node localization problems. in Proceedings of 1st ACM International Workshop on Wireless Sensor Networks and Applications, 112-121 (2002)

12. G Toussaint, The relative neighborhood graph of a finite planar set. Pattern Recognit. 12(4), 261-268 (1980). doi:10.1016/0031-3203(80)90066-7

13. G Mao, B Fidan, BDO Anderson, Wireless sensor network localization techniques. Comput Netw. 51(10), 2529-2553 (2007). doi:10.1016/j. comnet.2006.11.018

14. R Hach, Symmetric double sided two-way ranging. IEEE 802.15.4a standard, doc. IEEE P.802.15-05-0334-00-004a. (June 2005)

15. DB Johnson, DB Maltz, Dynamic Source Routing in Ad Hoc Wireless Networks. Mobile Computing (Kluwer Academic Publishers, Dordrecht. 153-181 (1996)

16. CE Perkins, EM Royer, Ad hoc on-demand distance vector routing. in Proceedings of IEEE MWCSA, LA, USA, 90-100 (Feb. 1999)

17. K Gabriel, R Sokal, A new statistical approach to geographic variation analysis. Syst Zool. 18, 259-278 (1969). doi:10.2307/2412323

18. nanoLOC TRX Transceiver (NA5TR1), Nanotron Technologies GmbH, Berlin, Germany, White paper NA-06-0230-0388-2.00, (April 2008)

19. Real Time Location Systems (RTLS), Nanotron Technologies GmbH, Berlin, Germany, White paper NA-06-0248-0391-1.02, (April 2007)

20. J Albowicz, A Chen, $L$ Zhang, Recursive position estimation in sensor networks. in Proceedings of 9th International Conference on Networking Protocols (ICNP), 35-41 (2001)

21. N Patwari, A A Hero, M Perkins, N Correal, B O'Dea, Relative location estimation in wireless sensor networks. IEEE Trans Signal Process. 51(8), 2137-2148 (2003). doi:10.1109/TSP.2003.814469

22. K Langendoen, N Reijers, Distributed localization in wireless sensor networks: a quantitative comparison. Comput Netw. 43(4), 499-518 (2003). doi:10.1016/S1389-1286(03)00356-6

23. J Cartigny, D Simplot, I Stojmenovic, Localized minimum-energy broadcasting in ad-hoc networks. in Proceedings of INFOCOM, San Francisco, USA, 2210-2217 (April 2003)

24. CK Seow, SY Tan, Localization of omni-directional mobile device in multipath environments. Progr Electromagn Res. 85, 323-348 (2008)

25. CS Tai, SY Tan, CK Seow, A robust non-line-of-sight localization system in an indoor environment. IEE Electron Lett. 46(8), 593-595 (2010). doi:10.1049/el.2010.2955

26. $\mathrm{PC}$ Chen, A non-line-of-sight error mitigation algorithm in location estimation. in Proceedings of International Conference on IEEE Wireless Communications and Networking, 1, 316-320 (1999)

27. MP Wylie, J Holzman, The non-line of sight problem in mobile location estimation. in Proceedings of 5th IEEE International Conference Universal Personal Communications. 2, 827-831 (1996)

doi:10.1186/1687-1499-2011-52

Cite this article as: Kong et al.: Localization and dynamic link detection for geographic routing in non-line-of-sight (NLOS) environments. EURASIP Journal on Wireless Communications and Networking 2011 2011:52. 